\title{
Fungal endocarditis in an extremely low birth weight infant. Case report
}

\author{
Abdurrahman A. Ozdemir, M.D. ${ }^{a}$, Tugce K. Oral, M.D. ${ }^{b}$ and Aydin Varol, M.D. ${ }^{a}$
}

\begin{abstract}
Survival rates of premature infants have increased with developing technology and evolving knowledge but lateonset sepsis is more frequent due to prolonged hospitalization periods of these infants.

We report the case of an extremely low birth weight premature infant who was admitted with respiratory distress syndrome, developed necrotizing enterocolitis and fungal sepsis with endocarditis by Candida albicans. He was treated with antifungal therapy until fungal vegetation resolved. Fungal sepsis and endocarditis may be an important morbidity in preterm infants; we review the recent literature about the risk factors, diagnosis, treatment and prevention of fungal sepsis in these high risk infants.

Key words: premature infant, candidiasis, endocarditis.

http:/ /dx.doi.org/10.5546/aap.2016.eng.e117
\end{abstract}

\section{INTRODUCTION}

Prolonged life expectancy with rapidly developing treatment options leads to increasing rate of sepsis of premature infants. Prematurity, immune deficiency, prolonged intubation, usage of central catheter lines, inadequate enteral feeding, longer duration of total parenteral nutrition and usage of broad spectrum antibiotics have been reported as the main risk factors for fungal infections. Fungal infections and especially Candida spp. cause serious problems in extremely low birth weight (ELBW) infants with high mortality and morbidity rates. ${ }^{1-6}$

Invasive fungal infection rates in neonatal intensive care units are reported as 1 to $2 \%$ whereas Candida and Aspergillus species are the

a. Department of Pediatrics, Division of Neonatology, Istanbul Medicine Hospital, Istanbul, Turkey.

b. Department of Pediatrics, Division of Neonatology, Kanuni Sultan Suleyman Training and Research Hospital, Istanbul, Turkey.

E-mail Address:

Abdurrahman A. Ozdemir, M.D.: avarozdemir@gmail.com

Funding: none.

Conflict of interest: none.

Received: 8-24-2015

Accepted: 10-26-2015 most common causes in these infants. ${ }^{1}$ However, the incidence of invasive candidiasis has been reported as high as 5.5-20\% in ELBW infants. ${ }^{2}$ Although Candida albicans is responsible for most of neonatal infections, in recent studies, Candida parapsilosis was reported as the second frequent species in neonates. ${ }^{1-3}$ Candida spp. may also cause focal infections such as pneumonia, meningitis, pyelonephritis, and endocarditis besides invasive candidiasis. ${ }^{1-5}$ The frequency and severity of Candida infection is inversely associated with gestational age and birth weight of preterm infants. ${ }^{1-3,6,7}$ The mortality rate of invasive fungal infections vary between $20 \%$ to $50 \%$, therefore it is important to screen all of the organ systems and also start the anti-fungal therapy as soon as possible. . $^{1,3}$,

Herein, we report a preterm infant who had fungal endocarditis.

\section{CASE REPORT}

A male infant with a birth weight of $850 \mathrm{~g}$ was born at 27th week of gestation by caesarean section from the second controlled pregnancy of a 29 year old mother. The baby was transferred to the Neonatal Intensive Care Unit, intubated and given early surfactant therapy for respiratory distress syndrome. An umbilical catheter was inserted and ampicillin and gentamicin were started, as sepsis could not be excluded. Enteral feeding started on $3^{\text {rd }}$ day. The echocardiography (ECHO) showed a small atrial septal defect (ASD). On the $7^{\text {th }}$ day of life, it was started vancomycin treatment as Staphylococcus epidermidis was identified in the blood culture.

On the $16^{\text {th }}$ day, his abdomen became distended and bloody stool was detected. He had thrombocytopenia and was diagnosed as necrotizing enterocolitis (NEC); enteral feeding was stopped and his treatment was changed. On the $22^{\text {nd }}$ day of hospitalization, general status was worsened. At that time, laboratory data showed leukocytes $29800 / \mathrm{mm}^{3}$, platelets $110000 / \mathrm{mm}^{3}$, and increased CRP $(56.9 \mathrm{mg} / \mathrm{dl})$ level. Although cerebrospinal fluid (CSF) and urine culture was sterile, Candida albicans was identified in his blood culture, the central catheter was removed 
and fluconazole was added to the treatment. The following cultures obtained at the 72 hours and first week were negatives. However, as C. albicans was again identified in the blood culture on the second week of the antifungal therapy, fluconazole treatment was switched to amphotericin B. Repeated cranial and abdominal ultrasounds were found normal but ECHO showed a mobile mass, $14 \mathrm{~mm}$ in length and $3 \mathrm{~mm}$ in width (vegetation, fungal ball?) atright atrium, passing through tricuspid valve and reaching to the inlet of right ventricle through ASD. (Figure 1)

In serial ECHOs, the vegetation showed regression so antifungal treatment was continued. A total of 10 blood cultures were obtained at the follow-up and as the last 3 blood cultures were sterile, amphotericin B treatment with duration of 7 weeks was discontinued after the disappearance of the vegetation on $\mathrm{ECHO}$ (Figure 2). The neurological examination of the infant, magnetic resonance imaging (MRI) and otoacoustic emission test response were all normal.

\section{DISCUSSION}

Late-onset sepsis frequency in premature infants is directly proportional with duration of hospitalization and inversely associated with gestational week and birth weight. ${ }^{1,2,5}$ Candida infections are among the most common cause of general hospital infections, with an incidence of $2.6-10 \%$ in very low birth weight (VLBW) and $5.5-20 \%$ in ELBW infants; the mortality rate is

FIGURE 1. The echocardiography showing the vegetation before treatment.

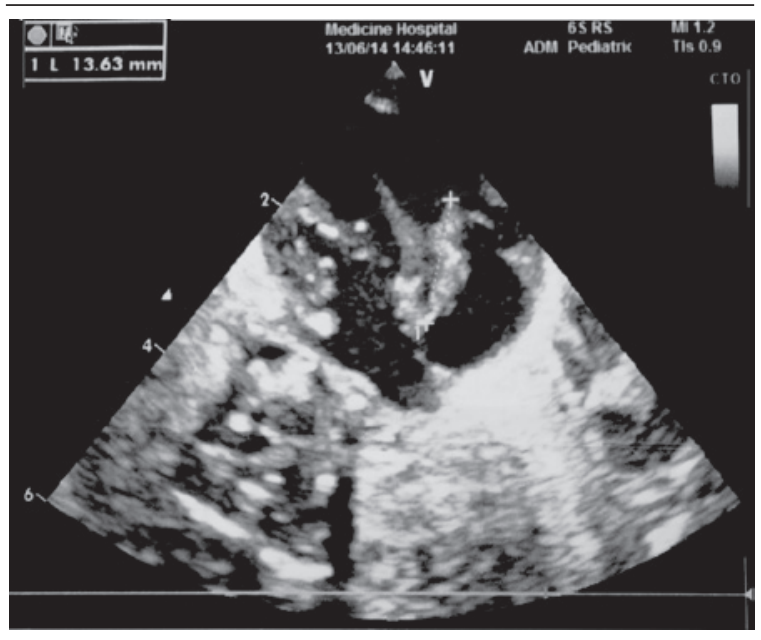

$20-50 \%{ }^{2,7,8}$ Fungal infections incidence was $0.5 \%$ in our NICU and we previously had no mortality due to fungal sepsis. The most common Candida types are C. albicans and C. parapsilosis. ${ }^{2,3}$ C. albicans constitutes more than $50 \%$ of neonatal fungal infections.$^{1,2}$ C. albicans was isolated in our case as consistent with the literature.

Most of the infants colonize with Candida spp. weeks after birth. ${ }^{1,4}$ After colonization, risk factors like prematurity, prolonged intubation, delay in enteral feeding, prolonged total parenteral feeding, broad spectrum antibiotic, steroid and $\mathrm{H} 2$ blocker usage, surgical interventions, gastrointestinal pathology such as NEC, and prolonged used of central catheter enhance the frequency of fungal infections. Therefore, these risk factors must be avoided and after detection of Candida infection, central catheter must be removed..$^{1-3,6,7}$ Risk factors such as prematurity, ELBW, inadequate enteral feeding, prolonged total parenteral feeding, prolonged use of central catheter, presence of sepsis and use of broad spectrum antibiotics were present in our case and central catheter was removed after the diagnosis of infection.

As a result of Candida colonization, mucocutaneous candidiasis, candidemia, catheter related infection, invasive and systemic infection may develop. Invasive infection of the blood, urine, cerebrospinal fluid (CSF) or peritoneal fluid can also lead to disseminated infection. ${ }^{1-4}$

Laboratory and clinical findings of invasive candida infection cannot be differentiated from bacterial sepsis. But the findings such as

FIGURE 2. The echocardiography showing the disappearance of vegetation after treatment.

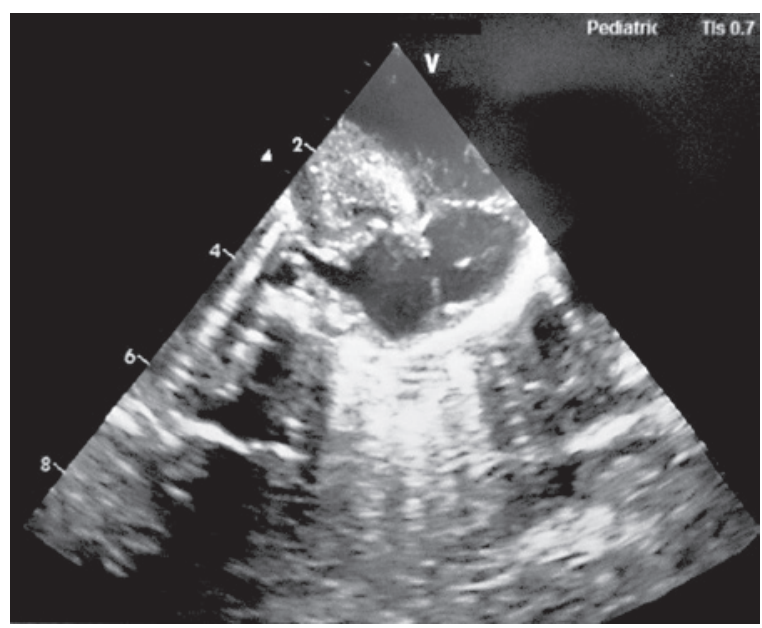


resistant hypoglycemia, thrombocytopenia and hypotension should be considered alarming. ${ }^{1-3}$ When infection is suspected, blood culture should be drawn and repeated in 48 hours. Candida must be detected in blood culture for definitive diagnosis. However, blood cultures have very low sensitivity for candidiasis. Tests such as anti-Candida antibody, beta-glucan antibody, d-arabinitol concentration, polymerase chain reaction (PCR) exist to determine Candida infection but not widely used..$^{1-4,8}$ The possibility of detecting in blood culture is $28-50 \%$ and we were able to detect it in our infant. ${ }^{4,5,7}$

Central nervous system (CNS), urinary system, eye, heart, liver involvements may be seen due to hematogenous spread..$^{1-5,9-12}$ Therefore, when candidemia is determined, ophtalmological examination, CSF and urine analysis, cranial and abdominal ultrasounds and ECHO must be performed..$^{2,3,9}$ Other organ involvement may accompany in premature infants with the rates of CNS involvement 10-64\%, renal involvement $5-33 \%$, and endophthalmitis $6 \%$., We performed tests on all systems in our case and detected endocarditis.

Cardiac involvement in systemic fungal infections is usually due to C. albicans. ${ }^{2-4}$ Endocarditis was reported in 5\% of invasive fungal infections with a mortality rate of over $60 \%$ in ELBW infants. ${ }^{2,410}$ Therefore, ECHO should be performed in all infants with Candida infections. In such cases, aggressive treatment should be started rapidly and should involve the removal of the vegetation besides the medical therapy. But as seen is this case, removal of vegetation may not be possible in extremely premature infants and longer duration of medical therapy may be needed. In addition to the previously mentioned risk factors, congenital heart disease might also be considered as a risk factor for the location of endocarditis in our case. . $^{1-4,9}$

Fluconazole, conventional amphotericin B, liposomal amphotericin B, flucytosine are used for treatment. Drugs like voriconazole, caspofungin and echinocandin also exist but researches are not enough for newborns. Duration of treatment is not certain but the recommendation is 2-6 weeks, depending on severity and widespread of the disease. ${ }^{1-3,10,13-15}$ In our case had C. albicans endocarditis which also resolved with systemic therapy. Treatment was started with fluconazole but switched to conventional amphotericin B as a result of inadequate response and lasted for 7 weeks.
During antifungal treatment, side effects like nephrotoxicity, hepatotoxicity, electrolyte imbalances may be seen. ${ }^{2}$ No side effect due to the therapy was observed in our case.

In prevention of candida infection, risk factors should be minimalized. Intravenous fluconazole may be used for 2-6 weeks in ELBW infants as prophylaxis ( $3 \mathrm{mg} / \mathrm{kg} /$ day, twice a week), starting the first day of life. ${ }^{14,15}$ We did not use prophylaxis in our case because the absence of previous fungal infection at our unit.

Neonatal candidiasis is associated with significant mortality (\%30-60) and morbidity in ELBW infants. ${ }^{3,4}$ Mental and psyhcomotor development are affected by the rate of $34-70 \%$ and hearing loss is seen especially in ELBW infants. For this reason, neurological follow-up is important. ${ }^{3}$ Otoacoustic emission test responses and cranial MRI were normal in our case.

In conclusion, Candida infections should be considered as a cause of both late-onset and very late-onset sepsis with existence of risk factors and prophylaxis for fungal infection considered in EBLW infants.

\section{REFERENCES:}

1. Hostetter MK. Fungal infections in the neonatal intensive care unit. En: Gleason CA, Devaskar SU, eds. Avery's diseases of the newborn. 9th ed. Philadelphia: Elsevier Saunders; 2012.Pgs.565-9.

2. BendelCM.Candidiasis. En:Remington JS, Klein JO, Wilson $\mathrm{CB}$, Nizet V, et al, eds. Infectious diseases of the fetus and newborn. 7th ed. Philadelphia: Elsevier Saunders; 2011. Pgs.1055-77.

3. Kaufman DA, Fairchild KD. Clinical microbiology of bacterial and fungal sepsis in very-low-birth-weight infants. Clin Microbiol Rev 2004;17(3):638-80.

4. Hundalani S, Pammi M. Invasive fungal infections in newborns and current management strategies. Expert Rev Anti Infect Ther 2013;11(7):709-21.

5. Steinbach WJ, Cohen-Wolkowicz M, Benjamin DK Jr. Principles of antifungal therapy. En: Kliegman RM, Stanton BM, St. Geme J, Schor N, et al, eds. Nelson textbook of pediatrics. 19th ed. Philadelphia: Elsevier Saunders; 2011. Pgs.1053.

6. Feja KN, Wu F, Roberts K, Loughrey M, et al. Risk factors for candidemia in critically ill infants: a matched casecontrol study. J Pediatr 2005;147(2):156-61.

7. Benjamin DK Jr, Stoll BJ, Gantz MG, Walsh MC, et al. Neonatal candidiasis: epidemiology, risk factors, and clinical judgment. Pediatrics 2010;126(4):e865-73.

8. Montagna MT, Lovero G, De Giglio O, Iatta R, et al. Invasive fungal infections in neonatal intensive care units of Southern Italy: a multicentre regional active surveillance (AURORA project). J Prev Med Hyg 2010;51(3):125-30.

9. Vricella LA, Khambadkone S, Yates R, Tsang VT. Right ventricular inflow obstruction from massive fungal vegetation presenting as neonatal circilatory collapse. Eur J Cardiothorac Surg 2003;24(2):323-4.

10. Cetin M, Ozen S, Yildırımer M, Deveci M, et al. Premature newborn with intracardiac fungus balls and endocarditis 
which is curable only with anti-fungal therapy: case presentation. Case Report in Clinical Medicine 2013;2(5):315-7.

11. Ben Ameur S, Hentati Y, Ben Dhaoui M, Weli M, et al. Candidose rénale chez le nouveau-né: à propos d'une observation. Arch Pediatr 2014;21(3):287-90.

12. Fernandez M, MoylettEH, Noyola DE, Baker CJ. Candidal meningitis in neonates: a 10 year rewiev. Clin Infect Dis 2000;31(2):458-63.

13. Azhar A. Successful management of fungal pericarditis and endocarditis in a neonate: a case report. J Saudi Heart Assoc 2012;24(3):195-9.

14. Botero-Calderon L, Benjamin DK Jr, Cohen-Wolkowiez M. Advances in the treatment of invasive neonatal candidiasis. Expert Opin Pharmacother 2015;16(7):1035-48.

15. Cetinkaya M, Ercan TE, Saglam OK, Buyukkale G, et al. Efficacy of prophylactic fluconazole therapy in decreasing the incidence of candida infections in extremely low birth weight preterm infants. Am J Perinatol 2014;31(12):1043-8. 